\title{
Modeling and Characterization of MTF and Spectral Response at Small Pitch on Mercury Cadmium Telluride
}

\author{
J. BERTHOZ, ${ }^{1,3}$ R. GRILLE,${ }^{1}$ L. RUBALDO,${ }^{1}$ O. GRAVRAND, ${ }^{2}$ \\ A. KERLAIN ${ }^{1}$ N. PERE-LAPERNE, ${ }^{1}$ L. MARTINEAU, ${ }^{1}$ \\ F. CHABUEL, ${ }^{1}$ and D. LECLERCQ ${ }^{1}$ \\ 1.-SOFRADIR, Avenue de la Vauve - CS20018, 91127 Palaiseau, France. 2.-CEA LETI- \\ MINATEC Campus, 17 rue des Martyrs, 38054 Grenoble Cedex 9, France. 3.-e-mail: jocelyn. \\ berthoz@sofradir.com
}

\begin{abstract}
Space applications are challenging infrared (IR) technologies, demanding the best system performance achievable. This requires covering the entire IR spectrum from short-wavelength infrared (SWIR) to very long-wavelength infrared (VLWIR) for various pixel sizes, which is possible thanks to a wellmastered mercury cadmium telluride technology. Because of its adjustable gap, it can be operated in all the IR bands. Nevertheless, technology optimization requires deep understanding of physical mechanisms. This paper presents computations by finite-element modeling of two aspects of electrooptical performance: spectral response and modulation transfer function (MTF). Computations and characterizations for all IR bands demonstrate the accuracy of our simulations and the state-of-the-art nature of our technology, which performs according to theory. This paper also highlights the capability to measure MTF at very small pitch $(10 \mu \mathrm{m})$ by a nondestructive method.
\end{abstract}

Key words: MTF, spectral response, HgCdTe, crosstalk, knife-edge method, HOT technology

\section{INTRODUCTION}

During the past few years, detector resolution improvement has generated a lot of interest. Since pixel pitch reduction improves resolution, the smallest pitch is desired. First, pixel pitch was reduced from $30 \mu \mathrm{m}$ to $15 \mu \mathrm{m} .{ }^{1}$ Now it is expected to achieve $12 \mu \mathrm{m}^{2}$ or $10 \mu \mathrm{m} .{ }^{3}$ Recently, the optimum pixel pitch was estimated to be $3 \mu \mathrm{m}$ for the midwavelength infrared (MWIR) band and $5 \mu \mathrm{m}$ for the long-wavelength infrared (LWIR) band. ${ }^{4}$

One of the key performance metrics for detector resolution is the modulation transfer function (MTF), which represents the ability of a system to distinguish contrast for different spatial frequencies. MTF optimization requires deep understanding of physical mechanisms. It has been shown previously that the ideal square pixel is not sufficient for predicting the MTF. ${ }^{5}$

(Received December 18, 2014; accepted May 18, 2015;

published online June 12, 2015)
For space applications, the spectral response is also a key figure of merit. Product specifications require precise control of its value at different wavelengths. Thus, it is mandatory to be able to simulate the MTF and spectral response.

This paper presents computations by finite-element modeling of the spectral response and MTF for the entire infrared band. Computations and characterizations for small-pitch and high-operabilitytemperature (HOT) components are presented for different pitches and cutoff wavelengths.

\section{EXPERIMENTAL PROCEDURES}

Spectral response measurements were carried out using a Fourier-transform infrared (FTIR) spectroscope in step-scan mode with resolution of $8 \mathrm{~cm}^{-1}$ and $F_{\#}=4.5$. The spectral response was obtained from the measured interferogram by strong NortonBeer apodization ${ }^{6}$ and Fourier transformation.

The MTF was measured using a knife-edge method. This approach has been used successfully 
in the past from $30 \mu \mathrm{m}$ to $15 \mu \mathrm{m}$ pitch. ${ }^{5}$ This is a nondestructive method for measuring the pixel MTF in two directions. The principle is to scan a pixel with an illuminated knife-edge source. ${ }^{7}$ The measured MTF can be obtained by Fourier transformation of the pixel response. However, because of the optical bench, the pixel MTF is convolved with the optical MTF as follows:

$$
\mathrm{MTF}_{\text {measured }}=\mathrm{MTF}_{\text {pixel }} \times \mathrm{MTF}_{\text {optical bench }} \text {. }
$$

To extract the pixel MTF from the measurement, deconvolution is mandatory. Deconvolution can be achieved as long as the pixel MTF is lower than the optical MTF. However, with pixel reduction, the pixel MTF becomes closer to the optical MTF. This effect involves two major issues:

- Complete understanding of the optical bench is necessary;

- The pixel MTF is very sensitive to noise coming from the measurement.

The optical MTF is measured by a reference sampling diode. This measurement can face the diffraction limit. For a circular aperture, the optical MTF at the diffraction limit can be expressed analytically as ${ }^{8}$

$$
\begin{aligned}
\mathrm{MTF}_{\text {diffraction }}= & \mid \frac{2}{\pi} \arccos (f \lambda F \#) \\
& -(f \lambda F \#)[1-(f \lambda F \#)]^{1 / 2} \mid,
\end{aligned}
$$

where $\lambda$ is the wavelength, $F_{\#}$ is the $F$-number, and $\mathrm{MTF}_{\text {diffraction }}$ is the diffraction-limited MTF.

Figure 1 presents a comparison between the measurement and the diffraction limit for wavelength of $3.8 \mu \mathrm{m}$ and $F_{\#}=2.9$.

As a consequence of aberrations present in the measurement, diffraction does not completely describe the optical-bench MTF. Aberrations have to be taken into account for the deconvolution. However, noise is added when the measurement is deconvoluted. Moreover, this noise is more critical for high spatial frequencies, where the optical MTF is close to zero. Thus, it is more problematic for

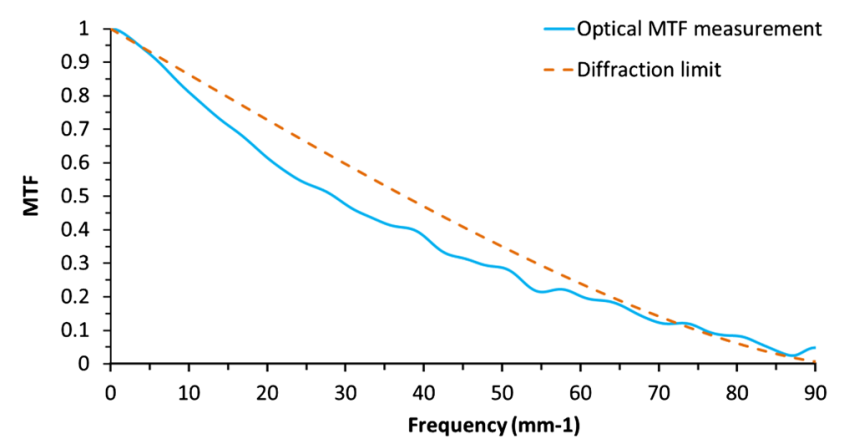

Fig. 1. Comparison between optical-bench MTF and the diffraction limit in the MWIR band with $\lambda=3.8 \mu \mathrm{m}$ and $F_{\#}=2.9$. small pitch. For these reasons, optical MTF modeling is necessary.

Aberration modeling is achievable with Zernike circle polynomial decomposition. ${ }^{9}$ Each polynomial represents a different aberration. Figure 2 shows the modeling and the measurement for the MWIR band. In this figure, 11 orders of Zernike polynomial are used.

There are different ways to perform the deconvolution. The easiest solution is to divide the measured MTF by the optical MTF. However, such division can amplify noise coming from the MTF measurement. This amplification is more important for high spatial frequencies where the optical MTF is close to zero. The method chosen for the deconvolution is the Richardson-Lucy algorithm, ${ }^{10}$ an iterative expectation-maximization deconvolution algorithm. It is applied in real space, implying no problem of divergence due to MTF division.

Instead of knife-edge illumination, a pinhole can also be used for spot-scan measurements. In this condition, an area of $3 \times 3$ diodes is scanned and the signal coming from the central pixel is collected. Using the same method of deconvolution, the MTF can be extracted with the central pixel response. In addition, by inverse Fourier transformation, the crosstalk can also be measured.

\section{COMPUTATION}

Computations were carried out using the finiteelement method (FEM). This method is currently used for MTF and spectral response computations. ${ }^{11-13}$ Two equations have to be solved: an optical equation and a semiconductor equation. With the optical equation, the photogeneration is computed. We use an analytic form for the photogeneration which does not include interference or diffraction. Indeed, with our FTM measurement, the optical wave is absorbed before any reflection in the component. For the spectral response, because of the thick substrate, the light loses its coherence and a ray-tracing method is sufficient. Thus, the photogeneration can be defined as

$$
G(x, y, z)=P(x, y) \times \Phi_{0} \times \alpha \times \mathrm{e}^{-\alpha z},
$$

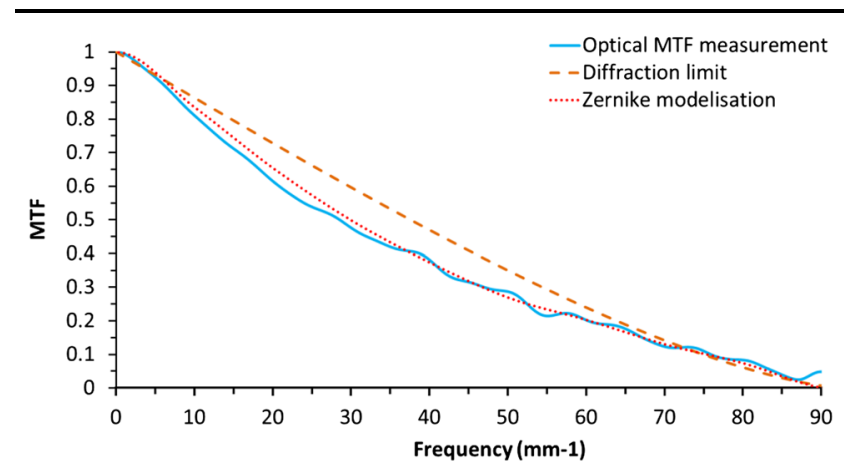

Fig. 2. Juxtaposition of the optical-bench MTF, the diffraction limit, and the Zernike modeling in the MWIR band. 
where $\alpha$ is the absorption coefficient, $z$ is the propagation wave axis, $\Phi$ is the incident photon flux, and $P$ is the optical profile. The absorption coefficient is taken from Moazzami. ${ }^{14}$ The form of the optical profile depends on the computation:

- For an MTF, the optical profile is a Heaviside function;

- For a spectral response, the optical profile equals 1 to have uniform illumination.

When the photogeneration is computed, the ambipolar drift equation has to be solved. This relation links the photogeneration to the minority carrier distribution as follows:

$$
\frac{n}{L_{\mathrm{d}}^{2}}+\vec{\nabla}[-\overrightarrow{\nabla n}]=\frac{G}{D}
$$

where $n$ is the minority carrier concentration, $L_{\mathrm{d}}$ is the diffusion length, and $D$ is the diffusion coefficient. $L_{\mathrm{d}}$ and $D$ are considered uniform throughout the whole computational domain. These values are obtained with specific devices and with quantum efficiency computation.

Finally, with the minority carrier concentration, it is possible to compute the photocurrent using the gradient around the diode junction area. ${ }^{15}$

Using this method, the MTF can be computed by solving these two equations for different positions of the optical profile. The obtained signal is used to compute the MTF by Fourier transformation. The spectral response can be computed directly with the solution of these equations for each wavelength.

\section{RESULTS AND DISCUSSION}

First, Figs. 3 and 4 show MTF measurements for different pitches and for two technologies: standard and HOT with cutoff wavelength of $5.2 \mu \mathrm{m}$ at $90 \mathrm{~K}$.

These figures show the very good accuracy of the computation. When the pitch is reduced, the MTF is always improved. However, at each Nyquist frequency, the MTF becomes lower with the pitch reduction. In this case, diodes are closer to each other, implying greater crosstalk. With the HOT technology, the MTF is slightly degraded. The reason is the larger lateral diffusion of carriers, implying higher electrical crosstalk. To verify this hypothesis, crosstalk measurements were carried out on these components, as shown in Fig. 5.

When the pitch is smaller or with the HOT technology, the crosstalk becomes greater. With this HOT technology, the diffusion length is larger due to a higher minority carrier lifetime. This explains the decreasing MTF value at Nyquist.

Then, we investigated the impact of the cutoff wavelength on the spectral response and MTF computations. Measurements and computations are compared. Figure 6 shows the measured and calculated spectral response from the SWIR to VLWIR.

Good agreement was found for the entire infrared band. Thanks to the fit, it is possible to extract the

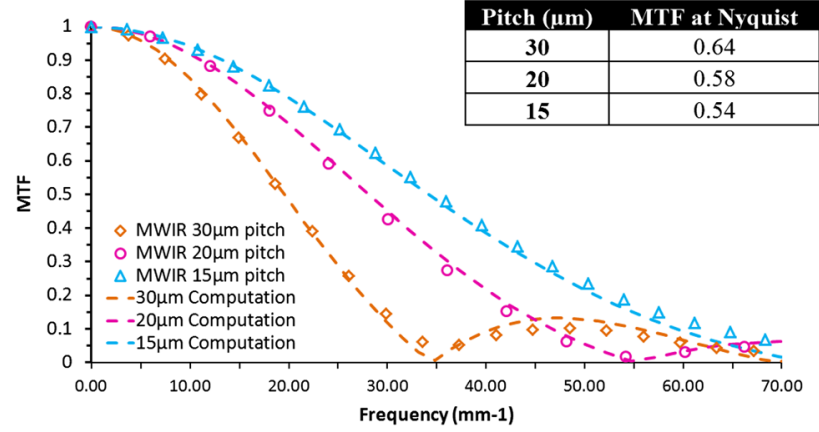

Fig. 3. MTF at different pitches in the MWIR band for standard technology at $90 \mathrm{~K}$.

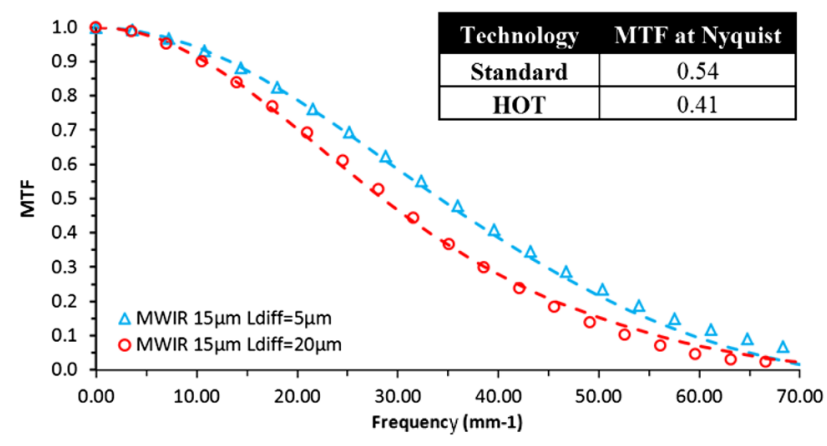

Fig. 4. MTF at different pitches in the MWIR band for HOT technology at $110 \mathrm{~K}$ and standard technology at $90 \mathrm{~K}$.

cadmium composition $x_{\mathrm{Cd}} \cdot x_{\mathrm{Cd}}$ is an important parameter because all the physical properties depend on it. These parameters will be used for MTF computations.

A comparison between the computations and measurements of the MTF at different cutoffs at $30 \mu \mathrm{m}$ pitch is shown in Fig. 7 .

The MTF computations and measurements were totally consistent. When the cutoff is larger, the MTF at Nyquist is also better. The reason is the larger diffusion length with the cutoff reduction. The MTF at Nyquist for the VLWIR band becomes higher than the ideal pixel. In this case, the pixel is not confined. The collection surface width $D$ is smaller than the pitch, and the diode array periodicity is then defined by this dimension (Fig. 8). Moreover, with the knife-edge method, only one pixel is sampled. Thus, the contribution of the sampling MTF is not included.

With a pixel not confined, the MTF at Nyquist and its first zero become higher. However, a lower collecting surface implies lower quantum efficiency. A trade-off has to be made to optimize these two performance features.

The same exercise was then carried out for the HOT technology at $15 \mu \mathrm{m}$ pitch (Fig. 9).

All the simulations as well as the measurements overlap. The MTF does not depend on the cutoff wavelength for the HOT technology. With the 
(a)

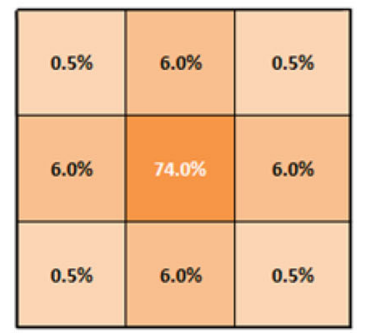

(b)

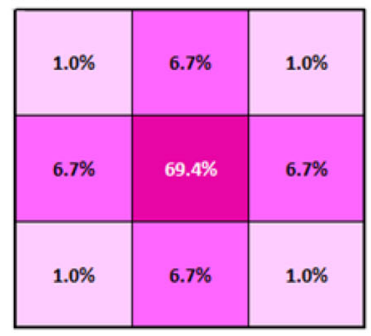

(c)

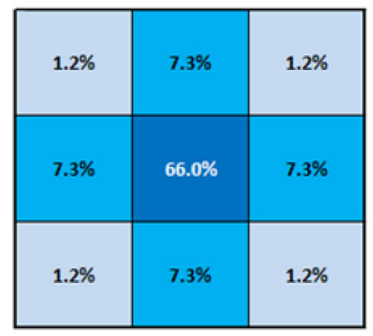

(d)

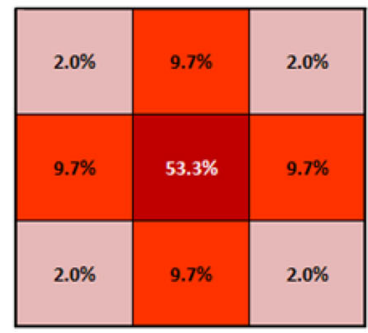

Fig. 5. Crosstalk measurements for different pitches and technologies in the MWIR band (a) $30 \mu \mathrm{m}$ pitch, (b) $20 \mu \mathrm{m}$ pitch, (c) $15 \mu \mathrm{m}$ pitch for standard technology, (d) $15 \mu \mathrm{m}$ pitch for HOT technology.

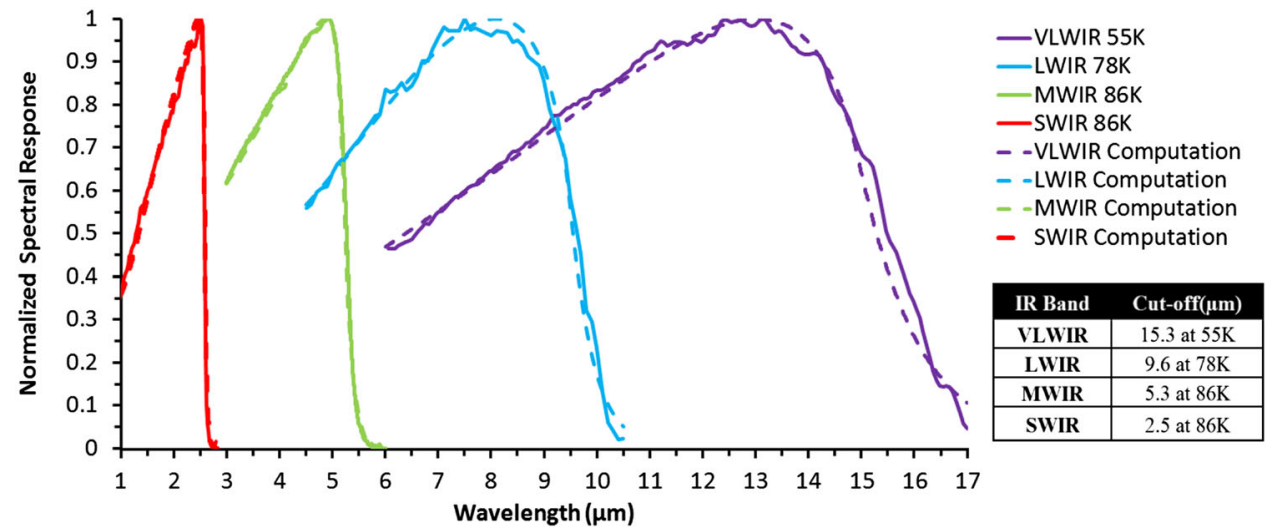

Fig. 6. Spectral response for different cutoffs.

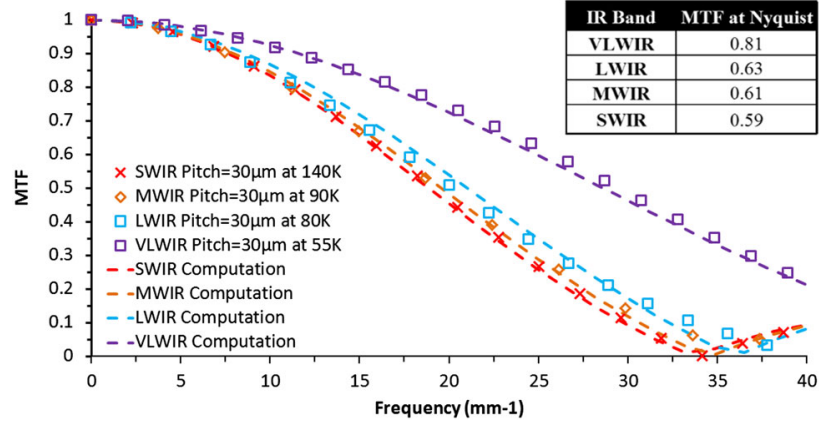

Fig. 7. Comparison between measurements and computations for different cutoffs at $30 \mu \mathrm{m}$ pitch.

standard technology, the MTF variation with the cutoff was due to the variation of the diffusion length. However, with the HOT technology at these temperatures, the pixel is self-confined. The diffusion length is greater than the pixel pitch for all the bands investigated, and thus the MTF is constant.

Finally, we investigated very small-pitch $(<15 \mu \mathrm{m})$ technologies. HOT technology at $12 \mu \mathrm{m}$ in the MWIR red band was studied. Figure 10 shows the MTF at $130 \mathrm{~K}$ compared with $15-\mu \mathrm{m}$-pitch technology.

The good agreement obtained between the measurement and computation highlights the capability of the MTF measurements and computations for small-pitch technology. As expected, the MTF increases with the pitch reduction.

The MTF was studied for $10-\mu \mathrm{m}$-pitch standard technology (based on $\mathrm{Hg}$ vacancies) in the MWIR red band for XGA format. This technology is well controlled at Sofradir, leading to very high-operability components. As illustrated in Fig. 11 by the perfect Gaussian shape of the noise-equivalent temperature difference (NETD) histogram, operability of $99.94 \%$ at $110 \mathrm{~K}$ and $F / 4$ was obtained with the standard criteria of NETD of $100 \%$, responsivity of $20 \%$, and DC level of $30 \%$.

An important effort has been made to optimize the photodiodes in order to reduce crosstalk and increase MTF values at the Nyquist frequency for this technology. FEM computation was an effective tool to optimize diode architectures.

Figure 12 compares the MTF at $10 \mu \mathrm{m}$ pitch for two different technologies.

The first developed technology had an MTF at Nyquist equal to 0.34 . Thanks to the computations, the diode architecture was optimized to obtain an MTF of 0.45 at the Nyquist frequency. Further improvements are currently ongoing. An optimized diode architecture with a $0.06 \mathrm{im}$ provement at Nyquist was identified and will be tested. 

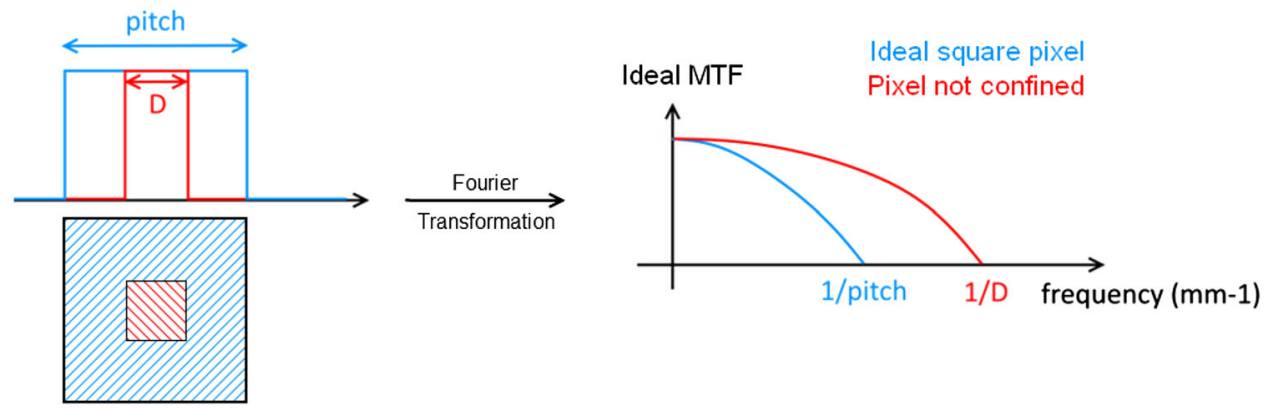

Fig. 8. MTF for an ideal square pixel and a pixel not confined.

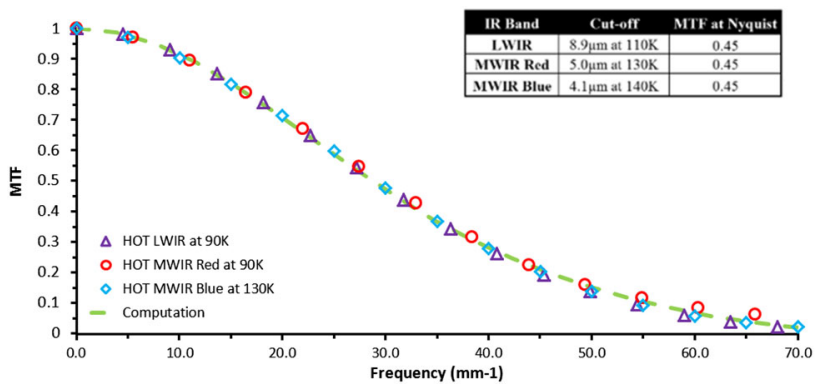

Fig. 9. MTF measurement and computation at $15 \mu \mathrm{m}$ for HOT technology for different cutoffs at $90 \mathrm{~K}$.

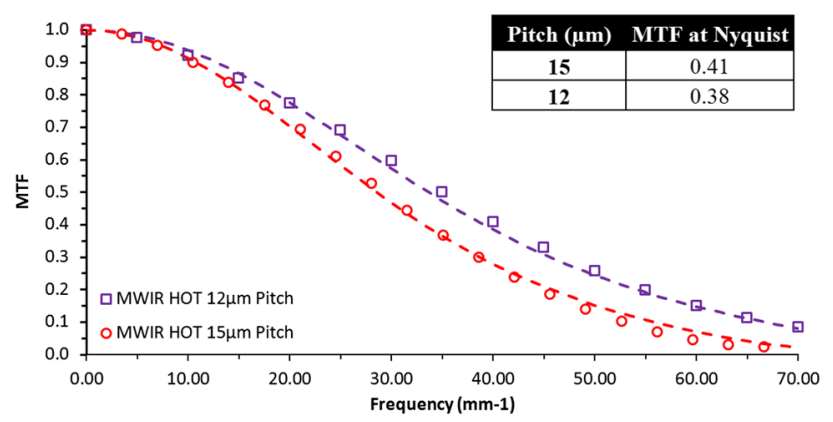

Fig. 10. MTF at $15 \mu \mathrm{m}$ and $12 \mu \mathrm{m}$ pitch for HOT technology in the MWIR band at $130 \mathrm{~K}$

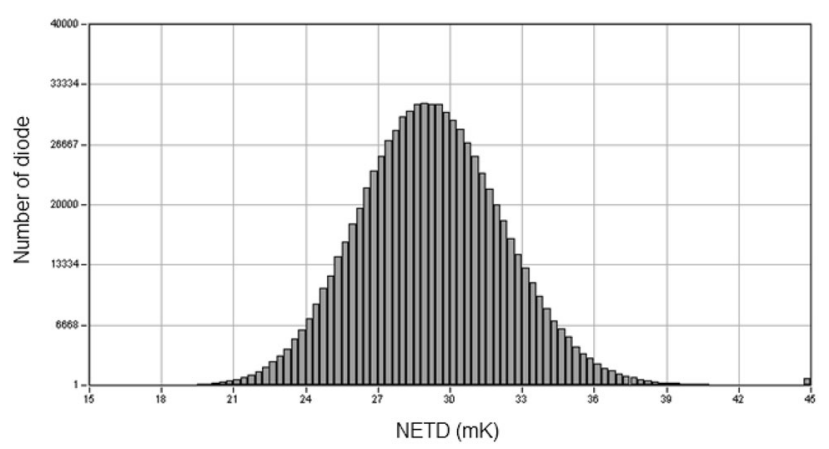

Fig. 11. NETD histogram at $110 \mathrm{~K}$ and $F / 4$.

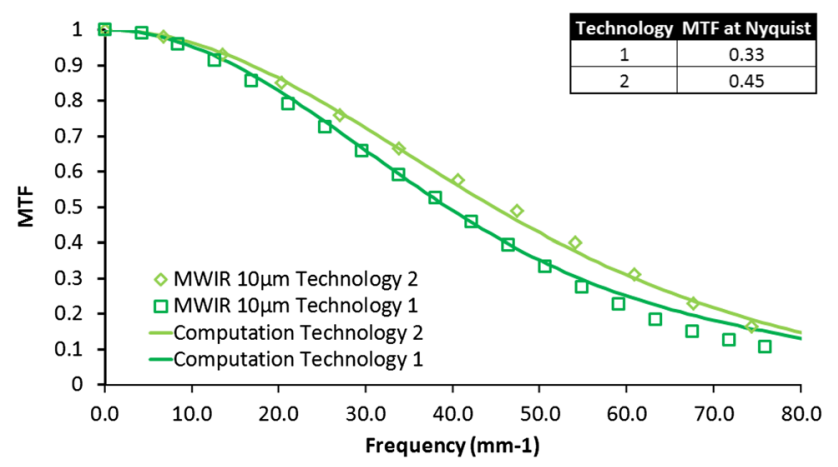

Fig. 12. MTF measurement for two technologies in the MWIR band.

\section{CONCLUSIONS}

A method for MTF measurement by a nondestructive technique is presented. To increase the accuracy of MTF measurements, we developed new measurement procedures and optical MTF modeling, and applied the Richardson-Lucy algorithm. This new MTF measurement protocol was successfully used for standard and HOT technologies with different cutoff wavelengths and pitches down to $12 \mu \mathrm{m}$ and $10 \mu \mathrm{m}$. A finite-element modeling program was developed to complete our measurement capabilities, and optimize the MTF. Clear MTF improvements were obtained for $10-\mu \mathrm{m}$-pitch $n$-on- $p$ technology with $\mathrm{Hg}$ vacancy doping. An MTF value of 0.45 as predicted by the computations was obtained experimentally, and further MTF improvements mandatory for HOT and small-pitch components are currently ongoing for all $\mathrm{HgCdTe}$ technologies developed at Sofradir.

\section{OPEN ACCESS}

This article is distributed under the terms of the Creative Commons Attribution 4.0 International License (http://creativecommons.org/licenses/by/4.0/ ), which permits unrestricted use, distribution, and reproduction in any medium, provided you give appropriate credit to the original author(s) and the source, provide a link to the Creative Commons license, and indicate if changes were made. 


\section{REFERENCES}

1. P. Castelein, F. Marion, J. Martin, J. Baylet, N. Moussy, A. Durand, J. Chamonal, and G. Destefanis, Proc. SPIE 5074, 52 (2003).

2. R. Strong, M. Kinch, and J. Armstrong, J. Electron. Mater. 42, 3103 (2013).

3. O. Gravrand, G. Destefanis, S. Bisotto, N. Baier, J. Rothman, L. Mollard, D. Brellier, L. Rubaldo, A. Kerlain, V. Destefanis, and M. Vuillermet, J. Electron. Mater. 42, 3349 (2013).

4. R.G. Driggers, Opt. Eng. 51, 063202 (2012).

5. J. Berthoz, L. Rubaldo, I.R. Grille, and O. Gravrand, 11th International Workshop on Low Temperature Electronics (WOLTE) (2014) pp. 5-7.

6. D.A. Naylor and M.K. Tahic, J. Opt. Soc. Am. 24, 3644 (2007).
7. G. Boreman, Modulation Transfer Function in Optical and Electro-Optical Systems (Bellingham: SPIE Press, 2001).

8. G. Boreman, Basic Electro-Optics for Electrical Engineers (Bellingham: SPIE Press, 1998).

9. J. Schmidt, Numerical Simulation of Optical Wave Propagation (Bellingham: SPIE Press, 2010).

10. W.H. Richardson, J. Opt. Soc. Am. 62, 55 (1972).

11. O. Gravrand and S. Gidon, J. Electron Devices 37, 1205 (2008).

12. B. Pinkie, J. Schuster, and E. Bellotti, Opt. Lett. 38, 2546 (2013).

13. T. Fishman, V. Nahum, E. Saguy, Z. Calahorra, I. Shtrichman, S.D. Scd, and P.O. Box, Proc. SPIE.

14. K. Moazzami, J. Phillips, D. Lee, S. Krishnamurthy, G. Benoit, Y. Fink, and T. Tiwald, J. Electron. Mater. 34, 773 (2005).

15. O. Gravrand, N. Baier, A. Ferron, F. Rochette, J. Berthoz, L. Rubaldo, and R. Cluzel, J. Electron. Mater. 43, 3025 (2014). 